\title{
Generalidades de Oestrus ovis: revisión bibliográfica
}

\author{
Ayala, M.J.; Flores, C. \\ Medicina Veterinaria, Facultad de Ciencias de la Salud \\ "Eugenio Espejo", Universidad UTE, Quito, Ecuador. \\ E-mail: josaayala28@gmail.com
}

\begin{abstract}
Resumen
Ayala, M.J.; Flores, C.: Generalidades de Oestrus ovis: revisión bibliográfica. Rev. Vet. 32: 2, 246-248, 2021. Oestrus ovis es el ectoparásito causante de la oestrosis, afectando a ovinos, caprinos y rara vez al hombre, invadiendo cavidades nasales, senos frontales y maxilares. Coloca sus larvas en ojos u hocicos, alimentándose de secreciones mucosas, para luego ser eliminadas mediante estornudos junto con las secreciones nasales. Llegando al suelo adquieren la forma de mosca. Los principales signos clínicos son el exudado nasal y los estornudos, así como sacudidas de la cabeza, ronquidos, prurito, intranquilidad y caminar en círculo, también llamado en "falso torneo". En ocasiones tienen fiebre. Para el tratamiento fue efectiva la aplicación subcutánea de ivermectina al $1 \%$ o closantel oral al $5 \%$ en dosis de $2 \mathrm{ml} / 10 \mathrm{~kg}$ de peso, logrando efectividad terapéutica en ovinos.
\end{abstract}

Palabras clave: ovinos, caprinos, ectoparásitos, moscas, cavidades nasales, ivermectina, closantel.

\begin{abstract}
Ayala, M.J.; Flores, C.: Overview of Oestrus ovis: bibliographic review. Rev. Vet. 32: 2, 246-248, 2021. Oestrus ovis is the cause of oestrosis, affecting sheep, goats, and rarely man, invading nasal cavities, frontal and maxillary sinuses, placing its larvae in eyes or snouts, feeding on mucous secretions, to later be eliminated by sneezing together with nasal secretions, reaching the ground where they will acquire the shape of a fly. The main clinical signs are nasal discharge and sneezing, others such as shaking the head, snoring, itching, restlessness, walking in a circle or also called "false tournament" and sometimes fever; For treatment, the subcutaneous application of $1 \%$ ivermectin or $5 \%$ oral closantel in a dose of $2 \mathrm{ml} /$ $10 \mathrm{~kg}$ of weight has been seen, achieving therapeutic effectiveness in sheep.
\end{abstract}

Key words: sheep, goats, ectoparasites, flies, nasal cavities, ivermectina, closantel.

\section{EL ECTOPARÁSITO}

Oestrus ovis es el ectoparásito causante de una enfermedad parasitaria conocida como oestrosis, afectando principalmente a rumiantes menores tales como ovinos, caprinos y -rara vez-al hombre, invadiendo partes de la cabeza como las cavidades nasales, senos frontales y maxilares ${ }^{7,10}$. Estos parásitos llegan a generar un impacto económico en la reproducción, existiendo un alto porcentaje de animales susceptibles en las regiones afectadas 7,10 .

El primer informe que demostró la existencia de este ectoparásito surgió en 1995, en el Municipio de Imias, Guantánamo (Cuba), y desde entonces llegaron más datos de provincias del medio oriente, demostrando que en el tiempo de registro (6 años: 2006-2011), la morbilidad promedio fue de $50,2 \%$, mientras que la mortalidad fue de $2,17 \%$ y $4,33 \%$ respectivamente ${ }^{7}$.

\section{INFECCIÓN}

Oestrus ovis es conocido por afectar principalmente ovejas y cabras, a quienes coloca larvas de la denominada "mosca del carnero" en ojos u hocicos de los animales que serán afectados. Las larvas se dirigirán a los senos paranasales, alimentándose de secreciones mucosas durante semanas o meses, siendo eliminadas junto con las secreciones nasales.

En el suelo se forman "pupas", las cuales al cabo de 3 a 6 semanas emergerán como adultos adquiriendo la forma de una mosca, asumiendo la habilidad de depositar rápidamente sus larvas en el hospedador, sin necesidad de interrumpir su vuelo; afectando no solo a los animales sino también a granjeros y personas indigentes de áreas rurales, sin que se den cuenta que fueron infectados ${ }^{6}$.

La mosca del carnero (Oestrus ovis) está distribuida universalmente. En estado adulto su morfología es 
de color oscuro, con manchas pequeñas en tórax y abdomen, que se encuentran cubiertos de vellosidades de un tono café oscuro y miden 10-13 $\mathrm{mm}^{\mathrm{de}}$ longitud ${ }^{2}$.

La infección se inicia al momento en que las moscas depositan las larvas en las cavidades nasales o cerca del hocico del animal, adhiriéndose hasta remontar rápidamente las fosas nasales, donde vivirán hasta completar su desarrollo larvario.

Así, la larva I se mantendrá hasta convertirse en larva $I I I$; para abandonar el hospedador desprendiéndose de la mucosa y provocando fuertes estornudos en el animal, entrando en contacto con el suelo, para así iniciar la metamorfosis, que dura de 2 a 8 semanas, y convertirse en pupa o crisálida, obteniendo la forma adulta que después de la cópula buscará las mucosas del hospedador ${ }^{8}$

El insecto adulto vive solamente alrededor de 15 días, ya que le es imposible alimentarse y su única fuente de nutrientes son las sustancias ingeridas en su estado larvario. La hembra adulta es capaz de depositar alrededor de 50 huevos (hasta 500 durante toda su vida), en los ollares de los hospedadores (ovinos) ${ }^{4}$.

\section{SIGNOS CLÍNICOS}

Los principales signos clínicos son el exudado nasal y los estornudos. Se revelan en los animales cuando tienen la cabeza mirando hacia el suelo, debido a la gran cantidad de exudado nasal. El ganado también muestra signos como sacudidas de la cabeza, ronquidos, prurito, intranquilidad, caminata en círculo (también llamada como "falso torneo") y en ocasiones, los animales pueden presentar fiebre ${ }^{1,5,9}$.

Al momento en que las larvas alcanzan los senos frontales y dañan el sistema nervioso central, puede ocurrir muerte de los ovinos y caprinos, luego de manifestar signos nerviosos (convulsiones, incoordinación motora, pérdida del equilibrio y ceguera, entre otros),

También existen afecciones adicionales a la oestrosis, incluyendo adenocarcinoma, neumonía intersticial, pleuroneumonía y abscesos pulmonares. Entre los signos que afectan económicamente a los carneros, cabe mencionar la pérdida del olfato, por lo cual ellos son incapaces de detectar a las hembras cuando están en estado de celo, las cuales no se preñan ${ }^{3,4,9}$.

\section{TRATAMIENTO}

Existen diferentes tipos de tratamientos con los cuales puede evitarse el contagio de Oestrus ovis. Uno de los principales métodos de control es el uso de antiparasitarios. En los animales infestados, se ha notificado que las lactonas macrocíclicas endectocidas son muy efectivas, debido a la elevada sensibilidad que tienen los miembros de Oestridae ${ }^{7}$.

Vía subcutánea, las aplicaciones de una formulación comercial de ivermectina al $\mathbf{1 \%}$ a razón de 200 $\mathrm{mcg} / \mathrm{kg}$ o closantel oral al $5 \%$ en una dosis de $2 \mathrm{ml} / 10$ $\mathrm{kg}$ de peso, pueden lograr un $100 \%$ de efectividad te- rapéutica en ovinos que estén infectados naturalmente por este agente ${ }^{7}$. Se recomienda el uso de tratamientos sistemáticos en el período en el que las moscas se encuentren en más actividad y en el comienzo del ciclo de hipobiosis, ya que éste parece ser muy eficaz en el control de Oestrus ovis.

Tal tratamiento garantiza no solo la eliminación de las larvas en los animales ya parasitados, sino que también influye en la dinámica poblacional de este insecto ya que disminuye la probabilidad de que se generen nuevas infestaciones en otros individuos y rebaños. Tales tratamientos pueden depender de las condiciones ambientales de cada región, ya que éstas van a influir en el número de ciclos que puede completar la "mosca del carnero" ${ }^{7}$.

\section{DAÑOS INFERIDOS}

En la última década, la enfermedad es reconocida como una parasitosis de gran importancia a nivel productivo. A partir de estudios realizados en Francia, esta afección está considerada como una enfermedad zoonótica y se la encuentra en numerosas áreas del mundo, especialmente en el mediterráneo europeo, en África y en Latinoamérica ${ }^{9}$.

La oestrosis es una afección vinculada exclusivamente con la ganadería extensiva ${ }^{5}$, en la cual los animales están en diario contacto con la naturaleza y es excepcional en las explotaciones en el régimen intensivo ${ }^{9}$. Se ha logrado comprobar que la infección que causa Oestrus ovis disminuye la rentabilidad de las explotaciones, ya que al reducir la ingesta de alimentos, conlleva a una reducción en la ganancia de peso en alrededor de un $16 \%$, disminución de lana en un $22 \%$ y de leche en un $10 \%{ }^{9}$

Esta parasitosis está condicionada fundamentalmente por factores climáticos considerados de gran importancia; como la temperatura, el fotoperíodo y el viento; aunque la temperatura llega a ser el factor determinante de la larviposicion. La humedad relativa y el fotoperíodo revelan influencias significativas sobre la prevalencia mensual ${ }^{9}$

La ostrosis es de presentación estacional en regiones con inviernos fríos y veranos cálidos, mientras que en los lugares donde tienen inviernos suaves y templados, pueden presentarse en cualquier época del año, por lo cual puede suceder en dos o tres generaciones ${ }^{9}$. El desarrollo en forma de larva de $O$. ovis puede ser interrumpido por un periodo seco, siendo esta una de las estrategias que pueden asegurar la perpetuación de $O$ ovis en las regiones donde las condiciones climáticas son más extremas.

\section{CONCLUSIÓN}

Se concluye que la enfermedad parasitaria causada por Oestrus ovis se puede controlar y prevenir con medidas adecuadas y correcta aplicación de medicamentos. De esta manera es factible reducir los índices de 
mortalidad que produce Oestrus ovis, logrando obtener un mejor control para la problemática del ganado ${ }^{6}$, tanto en el ámbito sanitario como en el reproductivo.

\section{REFERENCIAS}

1. Alem F, Kumsa B, Degefu H. 2010. Oestrus ovis larval myiasis among sheep and goats in Central Oromia, Ethiopia. Trop Anim Health Prod 42: 4, 697-703.

2. Beltrán M, Torres G, Segami H, Náquira C. 2006. Reporte de casos de miasis ocular por Oestrus ovis. Revista Perú Medicina, 1ra ed. [e-book], p.70.

3. Cepeda R et al. 2011. Ecobiology of the sheep nose bot fly (Oestrus ovis L.): a review. Rev MedVet 162: 11, 503-507.

4. Fernández R, Corrada R. 2010. Biodiversidad: inventario parasitológico. Acuario Nacional de Cuba, Ministerio de Ciencia, Tecnología y Medio. Ambiente, $38 \mathrm{p}$.
5. Fernández R. 2010. Oestrus ovis. Biodiversidad: inventario parasitológico. Acuario Nacional de Cuba, $38 \mathrm{p}$.

6. Mamani QP, Moreno LO. 2019. Oftalmo miasis por Oestrus ovis en un área urbana al sur del Perú. Rev Chil Infectol 36: 3, 384-386.

7. Matos V, Rodríguez J, Alfonso P, Olivares J. 2013. Oestrus ovis (Diptera: Oestridae): un importante ectoparásito de los ovinos en Cuba, 1ra ed., Rev SaudAnim, p.1.

8. Moreno V. 2016. Biología de Oestrus sp (Diptera: Oestridae). Estado de la cabra montés del parque natural de Sierra Nevada, ISBN: 9788416819577, págs. 15-17.

9. Petryna A. 2014. Oestrosis ovina y caprina: revisión bibliográfica. 1st ed. Director: Ginés Santiago de Gea, Río Cuarto, UNRC, Río Cuarto, Argentina, pp.51-52.

10. Puebla H, Zaldivar N, Soñora R. 2006. Oestrosis. aspectos biológicos de la miasis cavitaria ovina, 1ra. ed., Revista Electrónica de Veterinaria REDVET, pag. 2-3.

\title{
Asociación Cooperadora de la Facultad de Ciencias Veterinarias Universidad Nacional del Nordeste
}

Personería Jurídica $\mathrm{N}^{\circ} 647 / 92$ y $912 / 00$

\author{
Sargento Cabral 2139 \\ 3400 Corrientes \\ Argentina
}

La Asociación Cooperadora de la Facultad de Ciencias Veterinarias de la UNNE fue constituida el 10 de diciembre del año 1991 como entidad de bien público, con el objeto de promover y coadyuvar las actividades científicas, educativas y culturales relacionadas con las Ciencias Veterinarias. En tal sentido, implementa acciones para colaborar con la enseñanza, extensión, actualización y difusión científica que realiza dicha casa de estudios.

\section{Beneficios que brinda a sus asociados:}

- Fotocopias con descuentos especiales en la Fotocopiadora Copias.com que funciona dentro del predio de la Facultad de Ciencias Veterinarias.

- Descuentos para la adquisición de libros de distintas editoriales.

- Descuentos especiales en otros rubros.

Tel. (0379) 4425753 interno 186 • Página Web www.vet.unne.edu.ar • E-mail: cooperadora $@$ vet.unne.edu.ar 